\title{
CARACTERIZACIÓN TECNICO-PRODUCTIVA DE FINCAS LECHERAS DEL NORESTE DE LA PROVINCIA DE BUENOS AIRES, ARGENTINA
}

\author{
TECHNICAL-PRODUCTIVE CHARACTERIZATION OF DAIRY FARMS IN \\ THE NORTHEAST REGION OF BUENOS AIRES PROVINCE, ARGENTINA
}

\author{
Ricardo Lara ${ }^{1,2}$, Belén Lazzarini ${ }^{1}$, y Javier Baudracco ${ }^{1 *}$ \\ ${ }^{1}$ Facultad de Ciencias Agrarias, Universidad Nacional del Litoral, Kreder 2805, Esperanza, CP: \\ S3080HOF, Argentina. \\ ${ }^{2}$ Universidad Nacional de Luján, Ruta 5 y Av. Constitución, Luján, CP: 6700, Argentina. \\ * Autor para correspondencia: jbaudracco@yahoo.com
}

\section{RESUMEN}

La producción de leche en Argentina se intensificó, pero las fincas lecheras enfrentan limitantes para incrementar su productividad. El objetivo del presente trabajo fue detectar las principales limitantes a la producción de leche en las fincas lecheras de la región Noreste de la provincia de Buenos Aires, Argentina. Se confeccionó una guía para relevar datos productivos de 29 fincas lecheras. Las principales limitantes detectadas fueron: cantidad insuficiente de agua de bebida para el ganado (1 bebedero cada 19 ha) y de sombras naturales y artificiales $\left(1,8\right.$ y 2,9 m vaca-1, $^{-1}$ respectivamente); niveles deficientes de fósforo $\left(9,3 \mathrm{mg} \mathrm{kg}^{-1}\right)$ y sulfatos $\left(9,3 \mathrm{mg} \mathrm{kg}^{-1}\right)$ en el suelo; instalaciones de ordeño antiguas (21 años) y falta de inversión en equipos de ordeño; excesivo tiempo de ordeño $(3,3 \mathrm{~h}$ por ordeño); presencia de nitratos en el agua de bebida del ganado; y almacenamiento y distribución de efluentes inadecuado. Se infiere, a partir del análisis de correlaciones realizado, que las fincas que incrementaron la productividad a partir del incremento de la cantidad de vacas no incrementaron en similar medida su infraestructura. Las inversiones tendientes a superar las limitantes detectadas podrían permitir incrementos en la producción de leche y mejorar la sustentabilidad y rentabilidad del negocio lechero.

Palabras clave: Sistemas lecheros, producción de leche, relevamiento, limitantes, Argentina

\section{ABSTRACT}

Dairy production in Argentina has been intensified, but dairy farms are facing problems to increase productivity. This work aimed to identify the major constraints on milk production in dairy farms of the North East region of Buenos Aires province, Argentina. A survey was conducted to collect production data from 29 dairy farms. The major constraints were: insufficient water provision (there is one water trough every $19 \mathrm{ha}$ ), and insufficient natural and artificial shade for cows (1.8 and $2.9 \mathrm{~m}^{2}$ cow $^{-1}$, respectively); old dairy facilities (dating back 21 years) and lack of investment in milking equipment; long milking times ( 3.3 hours per milking); deficiencies in soil phosphorus (9.3 $\left.\mathrm{mg} \mathrm{kg}^{-1}\right)$ and sulphates $\left(11.7 \mathrm{mg} \mathrm{kg}^{-1}\right)$; presence of nitrates in cattle drinking water and inadequate effluent storage and distribution. According to the correlation analysis performed, it can be inferred that dairy farms that increased land productivity by increasing herd size did not improve farm infraestructure to meet new requirements. Investment to cope with the problems detected might increase milk production and improve the sustainability and profitability of dairy farms.

Key words: Dairy systems, milk production, constraints, Argentina, investment

Recibido: 18 enero 2019. Aceptado: 07 mayo 2019. 


\section{INTRODUCCIÓN}

La producción de leche es una actividad que permite el desarrollo económico y social de numerosas regiones del mundo. La producción de leche en Argentina se ha mantenido relativamente estable desde el año 1999, aproximadamente en 10.000 millones de litros anuales (OCLA, 2018). Sin embargo, la producción de leche se ha incrementado sustancialmente en países con sistemas de producción similar, pastoriles con suplementación, en los últimos años; así, por ejemplo en Uruguay el crecimiento fue $49 \%$ en los últimos 15 años (INALE, 2018), en Irlanda la producción creció 32\% desde 2009 a 2016 (OCLA, 2018), y en Nueva Zelanda se registró un crecimiento del $95 \%$ en los últimos 20 años (LICDairyNZ, 2018).

En Argentina, los niveles de producción de leche por vaca y la productividad anual por hectárea de una finca promedio son relativamente bajos en relación al potencial genético de los animales y a los alimentos utilizados, siendo la producción de leche individual de 17,5 litros vaca en ordeño ${ }^{-1}$ día $^{-1}$ y la productividad de 7.016 litros ha ${ }^{-1} \mathrm{año}^{-1}$, respectivamente (OCLA, 2018). Datos similares fueron publicados por Chimicz y Gambuzzi (2007) $\left(17,4\right.$ litros vaca en ordeño ${ }^{-1}$ día $^{-1}$ y 6.086 litros ha $\mathrm{a}^{-1}$ año $^{-1}$ ), lo que evidencia el estancamiento productivo de la lechería en Argentina, comprometiendo el resultado económico de las fincas y consecuentemente la sustentabilidad económica y social de la actividad lechera.

La cantidad de vacas por finca se incrementó en las últimas décadas en Argentina (Lazzarini et al., 2014; OCLA, 2018). Sin embargo, existen evidencias que muestran que el incremento en la cantidad de vacas por finca ocurrió sin la inversión necesaria en infraestructura (Baudracco et al., 2014), lo que afecta el bienestar de las vacas y las condiciones laborales de la gente. La falta de infraestructura y las condiciones laborales inadecuadas limitan la productividad de las fincas lecheras, generando una actividad poco atractiva (Lazzarini et al., 2019).

La provincia de Buenos Aires concentra el $22,1 \%$ de las fincas lecheras de Argentina, y dentro de esta provincia, el $42 \%$ (1.048 fincas) se encuentra en la cuenca Abasto de Buenos Aires (SENASA, 2018), ubicada en el Noreste de la provincia. Sin embargo, existe escasa información que describa la situación productiva de las fincas de dicha cuenca. El objetivo de este trabajo fue detectar los principales factores que podrían limitar la eficiencia de procesos productivos en fincas lecheras de la cuenca Abasto Buenos Aires, a través de un relevamiento de infraestructura, recursos naturales y tecnología aplicada.

\section{MATERIALES Y MÉTODOS}

Se realizó un relevamiento en 29 fincas lecheras ubicadas en la cuenca Abasto de Buenos Aires, entre las localidades de Luján (34⒊'13.0" S; 5906 $18.0^{\prime \prime}$ O) y Castelli ( $36^{\circ} 05^{\prime} 26^{\prime \prime}$ S; $57^{\circ} 48^{\prime} 30^{\prime \prime}$ O), y desde Navarro (3500'20.1" S; 59 $16^{\prime} 37.2^{\prime \prime}$ O) hasta la localidad de Lezama (35 $52^{\prime} 30.1^{\prime \prime}$ S; $57^{\circ} 53^{\prime} 49^{\prime \prime} \mathrm{O}$ ).

La región donde se realizó el relevamiento posee un clima templado húmedo con veranos muy cálidos y con precipitaciones anuales que rondan los $1.000 \mathrm{~mm}$. Los suelos son muy diversos, con capacidades de uso III y IV; presentan limitaciones de drenaje (baja infiltración) por presencia de horizonte B textural (arcilloso), siendo además una restricción importante el bajo contenido de fósforo, alrededor de $10 \mathrm{mg} \mathrm{kg}^{-1}$ (Sainz Rozas et al., 2012).

\section{Guía de relevamiento}

Se confeccionó una guía de relevamiento para recopilar la información en campo. Se relevaron aspectos productivos, de infraestructura, de manejo y tecnología aplicada y de recursos naturales de las fincas. La guía, con 333 ítems, se diseñó para maximizar la objetividad en la obtención de la información; por ello, se minimizó la cantidad de preguntas y se maximizó la cantidad de observaciones, priorizando parámetros cuantificables. Como ejemplo, para conocer la duración del ordeño se cronometró el tiempo entre el inicio y el fin del mismo, en lugar de interrogar al operario sobre su duración. Las variables que no pudieron ser cuantificadas, como por ejemplo "estado de los comederos" se describieron como variables cualitativas en una escala con tres opciones: bueno, regular y malo. En el transcurso de la visita se entrevistó al productor y a la persona encargada de la finca lechera. La duración total aproximada del relevamiento en el campo fue de 12 a 14 horas por finca, en una o dos visitas, y estuvo a cargo de un Ingeniero Agrónomo. La guía se construyó en base a variables definidas en un relevamiento previo (Baudracco et al., 2014).

\section{Características generales del establecimiento}

Mediante una entrevista al productor se registró la cantidad de vacas en ordeño (VO) y vacas secas (VS) promedio en el año, la producción anual de leche, la superficie destinada al rebaño de vacas totales (VT) (ordeño y secas), el régimen de tenencia de la tierra (propia o arrendada), la ocupación de los lotes destinados a VT (superficie de praderas, de cultivos anuales, superficie improductiva), la raza utilizada, los porcentajes de mortandad (de vacas y de terneros), la capacidad de uso de los 
suelos, la cantidad de reservas forrajeras y de concentrados comprados, los rendimientos de los cultivos y la tecnología utilizada en los mismos (genética, agroquímicos y manejo).

\section{Infraestructura relevada}

Se relevaron las instalaciones de ordeño, la sombra disponible para los animales, la cantidad y estado de las aguadas para los animales, el estado de los caminos internos para circulación de animales (callejones) y la infraestructura disponible para alimentación.

Instalaciones de ordeño. Se relevaron datos sobre la antigüedad de las instalaciones, tipo de instalación, cantidad de unidades de ordeño, el tipo y dimensionamiento del corral de espera, el sistema de refrigeración de los animales en la sala de ordeño y en el corral de espera, y el equipamiento disponible para el ordeño.

Sombras, aguadas y callejones. Se relevó el dimensionamiento de las sombras naturales (árboles) y artificiales, su orientación y el estado de las mismas. También se registró el acceso a aguadas y a comederos cercanos al área de sombras. Se relevaron todas las aguadas utilizadas por las vacas, en cantidad, capacidad, estado del piso circundante a la aguada y la distancia de las aguadas a las parcelas de pastoreo. Se relevaron los callejones, registrando pendiente, ancho y la cantidad de sitios con encharcamiento y pozos.

\section{Manejo y tecnología aplicada}

Crianza de terneros. Se relevó el sistema de crianza, el tiempo de permanencia del ternero con la vaca, tipo, cantidad y horarios de suministro de alimentos, el plan sanitario, limpieza de los comederos, criterio de destete y presencia/ ausencia de sombra en el sector de crianza.

Ordeño. Se relevó la duración, horarios y rutina de ordeño y la frecuencia de servicio de mantenimiento de la máquina ordeñadora.

Reproducción. Se relevó el tipo de servicio (natural o artificial), la disponibilidad de registros reproductivos, la distribución de partos y el sistema de detección de celos.

Cultivos. Se relevaron datos relativos a la tecnología y el manejo utilizado en los cultivos, tales como: cultivares utilizados, agroquímicos, fertilización (tipo, momento y dosis).

Alimentación. Se relevó la disponibilidad y estado de los comederos y la rutina de alimentación. En el caso de haber más de un rebaño de vacas en ordeño, toda la sección de alimentación se completó únicamente para el rebaño más grande de vacas en ordeño.

\section{Recursos naturales}

Suelo. Se tomaron 30 muestras por parcela, a $25 \mathrm{~cm}$ de profundidad, luego se realizó una muestra compuesta y se envió a laboratorio para análisis de materia orgánica (\%) por el método de Walkley y Black's (Jackson, 1982), pH en una relación suelo:agua de 1:2,5, nitrógeno $\left(\mathrm{NO}_{3}\right)$ por colorimetría, fósforo extractable por colorimetría por el método de Bray y Kurtz (Jackson, 1982), y azufre $\left(\mathrm{SO}_{4}\right)$ por turbidimetría (Jackson, 1982). En cada finca se tomó una muestra, en una parcela con pastura con más de dos años desde la implantación, que no haya sido utilizada para encierre de animales en los últimos tres años.

Agua de bebida para el ganado. Se tomó una muestra en la primera aguada que se encontró a la salida de la sala de ordeño, directamente desde el tanque o bebedero, para representar el agua que efectivamente consumen los animales; luego se llevó al laboratorio para determinar los siguientes parámetros: $\mathrm{pH}$ por potenciometría, concentración en $\mathrm{mg} \mathrm{kg}^{-1}$ de bicarbonatos, carbonatos, sulfatos, nitratos, sales totales por complejometría, sodio por fotometría de llama, y cloruros por colorimetría.

\section{Litros libres de alimentación}

Con los datos relativos a alimentación, y con el acceso a registros económicos de las fincas, se calculó la variable litros libres de alimentación por hectárea (LLA ha ${ }^{-1}$ año $^{-1}$ ) para cada una de las fincas. Esta variable representa el ingreso equivalente en litros de leche luego de descontar los costos de alimentación (concentrados, reservas y pasturas) y se calcula de la siguiente manera: LLA ha $^{-1}$ año $^{-1}=\left[\left(\right.\right.$ Ingreso por venta de leche $\mathrm{VT}^{-1}$ año ${ }^{-1}-$ Costo de alimentación $\mathrm{VT}^{-1}$ año $\left.^{-1}\right) \times \mathrm{VT} \mathrm{ha}^{1}$ ] $\div$ Precio por litro de leche

Este indicador está fuertemente relacionado a la eficiencia alimenticia y al resultado económico en fincas lecheras (Adduci et al., 2015).

\section{Análisis de los datos}

Los resultados de cada finca fueron examinados individualmente para detectar valores aberrantes. En caso de detectar valores aberrantes, se consultó nuevamente con el dueño o encargado de la finca para corroborar y corregir la información obtenida. Se utilizó estadística descriptiva; se estimó el promedio, desvío estándar y los valores máximos (MAX) y mínimos (MIN) para cada uno de los parámetros relevados. Se estimaron coeficientes de correlación (Pearson con nivel de significancia $\mathrm{p} \leq 0,05$ y $\mathrm{p} \leq 0,01)$ entre todas variables.

\section{RESULTADOS Y DISCUSIÓN}

\section{Características generales de la finca lechera promedio}

Las características generales de la finca lechera 
promedio se presentan en la Tabla 1. El sistema de producción típico es pastoril con suplementación durante todo el año, siendo la pastura de alfalfa (Medicago sativa L.) el principal cultivo, el cual ocupa $46 \%( \pm 17,4)$ de la superficie total. La finca promedio tiene $351( \pm 191)$ VT en una superficie de 215 ( \pm 119$)$ ha, equivalente a una carga animal de 1,64 $( \pm 0,41) \mathrm{VT} \mathrm{ha}^{-1}$, incluyendo la superficie improductiva $(14,5 \%$; definida como superficie no utilizada con cultivos). Si bien la carga animal es mayor al promedio del país, existe evidencia sobre la conveniencia económica de incrementarla aún más en los sistemas lecheros argentinos (Baudracco et al., 2017), siempre y cuando se cuente con la infraestructura adecuada.

La producción individual de leche fue de 23,9 litros VO día ${ }^{-1}$ y la productividad de 12.230 $\mathrm{L} \mathrm{ha}^{-1} \mathrm{año}^{-1}$ (Tabla 1), 37\% y $74 \%$ superior al promedio de Argentina, respectivamente. Todas las fincas utilizan vacas de raza Holstein, de aproximadamente $600 \mathrm{~kg}$ de peso vivo en los rodeos. El 100\% de las fincas relevadas cuentan con asesoramiento veterinario, el $89 \%$ con asesoramiento agronómico, y casi la mitad recibe asesoramiento adicional en nutrición animal.

\section{Infraestructura en la finca lechera}

Instalaciones de ordeño. En la Tabla 2 se observan datos sobre la infraestructura relevada en las fincas lecheras de la región noreste de la provincia de Buenos Aires. La totalidad de las fincas tiene una sala de ordeño tipo espina de pescado, con un promedio de 15 unidades de ordeño. En promedio, se reveló una antigüedad de $21( \pm 14)$ y $9( \pm 7)$ años para la instalación y la máquina de ordeño, respectivamente. Estos datos evidencian la falta de inversión en instalaciones y equipamiento de ordeño, lo que impone restricciones al bienestar animal, pudiendo predisponer a mastitis, y consecuentemente disminuir la producción de leche (Bareille et al., 2003). Resultados similares se observaron para una muestra de 190 fincas lecheras en la región pampeana de Argentina donde se observó que el $60 \%$ de las fincas presentan instalaciones de ordeño que superan o están próximas a cumplir su vida útil (INTA, 2018).

Respecto al corral de espera, todas las fincas lecheras tienen corral de cemento, con una superficie promedio de 1,6 $( \pm 0,9) \mathrm{m}^{2} \mathrm{VO}^{-1}$ para el rebaño más grande, valor adecuado para vacas Holstein de $550 \mathrm{~kg}$ (Chesterton et al., 1989). Sin embargo, el $34 \%$ tiene menos de $1,2 \mathrm{~m}^{2} \mathrm{VO}^{-1}$, lo que podría incrementar la predisposición a afecciones podales y lesiones entre animales por el reducido espacio. Dentro de la sala de ordeño, el $34 \%$ de las instalaciones cuenta con ventiladores, el 93\% tiene automatizada la alimentación con alimento concentrado, y el $34 \%$ posee extractores automáticos de pezoneras.

Respecto a la infraestructura para almacenamiento y tratamiento de efluentes, solo $14 \%$ de las fincas lecheras almacena y distribuye los efluentes, aunque la mayoría de los productores encuestados manifestó preocupación acerca de la problemática de los efluentes generados por la actividad.

Sombra para animales. La vaca lechera es sensible al estrés calórico, el cual genera disminución del consumo de alimentos y de la producción de leche (Flamenbaum y Gallon., 2010). La provisión de sombras naturales o

Tabla 1. Características generales de las fincas lecheras relevadas de la región noreste de la provincia de Buenos Aires, Argentina: uso de la superficie, cantidad de vacas y producción de leche (n = 29)

Table 1. General description of surveyed dairy farms in the North East region of Buenos Aires province, Argentina: land use, cattle stock, and milk production $(n=29)$

\begin{tabular}{lcccc}
\hline Variable & Promedio & Desvío standard & MAX $^{\mathbf{1}}$ & MIN $^{2}$ \\
\hline Vacas ordeño (VO) & 298 & 167,2 & 684 & 103 \\
Vacas totales (VT) & 351 & 190,9 & 774 & 126 \\
Superficie VT, ha & 215 & 119,3 & 535 & 71 \\
Superficie propia, \% & 78 & 38,6 & 100 & 0 \\
Producción por vaca, L VO-1 día $^{-1}$ & 23,9 & 2,83 & 29,1 & 18,3 \\
Producción diaria total, L día & 7.143 & $4.226,7$ & 16.384 & 2.782 \\
Productividad anual, L ha-1 año-1 $^{-1}$ & 12.230 & $3.782,3$ & 21.926 & 6.054 \\
Superficie de alfalfa, \% & 46 & 17,4 & 86 & 6 \\
Superficie de maíz, \% & 29 & 16,0 & 62 & 0 \\
Superficie de verdeo invierno, \% & 25 & 11,9 & 52 & 7 \\
\hline
\end{tabular}

${ }^{1}$ MAX: Valor máximo; ${ }^{2}$ MIN: Valor mínimo. 
Tabla 2. Descripción de la infraestructura relevada en las fincas lecheras de la región noreste de la provincia de Buenos Aires, Argentina: instalación de ordeño, sombras, aguadas, callejones.

Table 2. Description of the infrastructure in the surveyed dairy farms in the North East region of Buenos Aires province, Argentina: dairy facilities, shades, water troughs, cow lanes.

\begin{tabular}{|c|c|c|c|c|}
\hline & Promedio & Desvío standard & MAX & MIN \\
\hline \multicolumn{5}{|l|}{ Instalación de ordeño } \\
\hline Antigüedad instalación, años & 21 & 13,7 & 55 & 1 \\
\hline Unidades de ordeño, $\mathrm{n}$ & 15 & 6,1 & 28 & 8 \\
\hline Antigüedad máquina de ordeñar, años & 9 & 7,2 & 30 & 1 \\
\hline Antigüedad del equipo de frío, años & 9 & 15,4 & 80 & 1 \\
\hline Capacidad del equipo de frío, L & 10.861 & 6570,7 & 27.000 & 4.200 \\
\hline \multicolumn{5}{|l|}{ Sombras (dimensionamiento) } \\
\hline Sombra natural, $\mathrm{m}^{2} \mathrm{VO}^{-1}$ & 1,8 & 1,24 & 4,8 & 0,2 \\
\hline Sombra artificial, $\mathrm{m}^{2} \mathrm{VO}^{-1}$ & 2,9 & 8,17 & 8,7 & 0,4 \\
\hline \multicolumn{5}{|l|}{ Aguadas } \\
\hline Distancia prom. a aguada desde potrero, $(\mathrm{m})^{1}$ & 244 & 82,4 & 419 & 40 \\
\hline Relación superficie/aguadas, ha aguada ${ }^{-1}$ & 19 & 9,0 & 42 & 8 \\
\hline \multicolumn{5}{|l|}{ Callejones } \\
\hline Sitios dañados, cantidad ${ }^{3}$ & 2 & 1,1 & 4 & 0 \\
\hline Callejones en buen estado, $\%^{4}$ & 64 & 35,2 & 100 & 0 \\
\hline Callejones en estado regular, $\%^{4}$ & 28 & 30,4 & 83 & 0 \\
\hline Callejones en mal estado, $\%{ }^{4}$ & 8 & 18,7 & 67 & 0 \\
\hline
\end{tabular}

${ }^{1}$ Recorrido promedio de las vacas desde el centro del potrero hasta la aguada más cercana.

${ }^{2}$ Capacidad total de almacenamiento de agua, no necesariamente las vacas pueden acceder a toda el agua.

${ }^{3}$ Sectores con charcos de agua, desniveles pronunciados, pozos, etc.

${ }^{4}$ Apreciación visual.

artificiales en sistemas de producción en pastoreo es imprescindible para mitigar los efectos del estrés calórico en regiones como la relevada en el presente estudio. El estrés calórico es una situación frecuente en la región relevada. El índice de temperatura y humedad (ITH) es un índice de confort del ganado lechero que se utiliza para monitorear las condiciones que causan estrés térmico combinando la humedad relativa del aire $(\mathrm{HR} \%)$ y la temperatura del aire $\left(\mathrm{T}^{\circ}\right)$, y se calcula como (Dikmen and Hansen 2009):

$\mathrm{ITH}=\left(1,8 \times \mathrm{T}^{\circ}+32\right)-(0,55-0,55 \times \mathrm{HR} \% / 100)$ $\mathrm{x}\left(1,8 \times \mathrm{T}^{\circ}-26\right)$.

La producción de leche y el consumo de alimentos disminuye cuando el ITH alcanza el valor de 72 (Cerqueira et al., 2016). En la región relevada, en los meses de verano se registran aproximadamente ocho horas diarias con valores de ITH superiores a 72 .

En la Tabla 2 se observan detalles sobre la provisión de sombra natural y artificial en las fincas relevadas. A pesar de que el $100 \%$ de las fincas cuenta con sombra natural, la sombra natural disponible es de $1,8 \mathrm{~m}^{2}$ vaca $^{-1}$, lo cual es muy inferior al óptimo recomendado de $4 \mathrm{~m}^{2}$ vaca $^{-1}$ (Veissier, 2018). Considerando ambos tipos de sombra, solo el $38 \%$ de las fincas posee niveles adecuados de sombra $\left(4 \mathrm{~m}^{2}\right.$ vaca $\left.^{-1}\right)$ para mitigar los efectos del estrés calórico. El 34\% de las fincas presenta valores menores a $2 \mathrm{~m}^{2}$ vaca $^{-1}$. Estos resultados muestran que la cantidad de sombra disponible para los animales en la región noreste de la provincia de Buenos Aires es escasa, lo cual podría afectar el bienestar animal y la producción de leche en los meses calurosos. La disponibilidad de agua y comederos en los sectores de sombra también presenta carencias. La mitad de las fincas no tiene aguadas en el sector cercano a la sombra y solo un $38 \%$ tiene comederos en esa área.

Aguadas. Otro de los aspectos críticos detectados en el relevamiento fue la disponibilidad de agua de bebida para las vacas. En promedio las fincas relevadas tienen 1 aguada cada $19( \pm 9)$ ha. La escasa disponibilidad de aguadas obliga a las vacas a caminar largas distancias para acceder al agua mientras se encuentran en pastoreo, con una consecuente disminución de consumo de agua y de alimentos. Las vacas en pastoreo no deberían caminar más de $200 \mathrm{~m}$ para acceder al agua; distancias mayores afectan el patrón de utilización de las pasturas y reducen la eficiencia de cosecha (Miglierina et al., 2018), disminuyendo su consumo y consecuentemente la producción animal. El resultado del relevamiento indica que 
las vacas deben caminar en promedio $244( \pm 82) \mathrm{m}$ desde el centro del potrero hasta la aguada, con distancias superiores a los $200 \mathrm{~m}$ en el $48 \%$ de los casos.

Callejones. El 64\% de los callejones fue descrito como "buen estado", el restante $36 \%$ en estado "regular o malo". El $49 \%$ de los callejones se registró con pendiente adecuada, lo que favorece el escurrimiento de agua de lluvia y permite un secado más rápido de los mismos, evitando posibles problemas podales y permitiendo que las ubres lleguen más limpias al ordeño, reduciendo el tiempo empleado en la rutina de ordeño. EL $51 \%$ fue descrito como plano o hundido, lo cual puede predisponer a problemas podales y de ubre (Lawrence et al., 2011).

\section{Manejo y tecnología aplicada}

Crianza de terneros. El porcentaje de mortandad de terneros en la crianza (desde nacimiento hasta los 60 días) fue de 7,9\%, lo cual es inferior a la mortandad promedio de las fincas lecheras de Argentina, aproximadamente 11,5\% (INTA, 2018), pero superior a valores reportados en otros países, tales como Suecia, Estados Unidos y Dinamarca (Torsein, 2011). La alta mortandad de terneros es una causa importante de pérdida económica en la producción lechera (Mee, 2008). Las fincas lecheras argentinas tienen dificultad para crecer en cantidad de vacas, más aún, el stock nacional lechero decreció en los últimos años (OCLA, 2018). Una de las razones por las cuales se dificulta el crecimiento del stock de ganado lechero argentino es el alto porcentaje de mortandad durante el periodo de crianza. La evidencia de relevamientos exhaustivos previos en fincas lecheras muestra que la alta mortandad se debe principalmente a falencias en el proceso de calostrado, la falta de infraestructura y la falta de capacitación del personal a cargo (Baudracco et al., 2014).

En un $61 \%$ de las fincas relevadas se utiliza un sistema de crianza individual de terneros. En el $36 \%$ de los casos los terneros se crían en forma individual, con el ternero atado con cadena a un alambre, sujeta al piso, de cuatro metros de largo, lo que le permite mayor desplazamiento. En un 3\% de los casos relevados se crían teneros en forma grupal o crianza colectiva, sin cadenas.

En promedio se ofrecen 4,3 $\mathrm{L}$ de leche diarios por ternero durante los 60 días de crianza en las fincas relevadas. Este volumen de ingesta de leche es insuficiente, ya que las terneras que se alimentan con leche deberían ingerir aproximadamente el $10 \%$ de su peso vivo (Khan et al., 2011), y a las dos o tres semanas de vida, $\mathrm{su}$ peso es superior a los $50 \mathrm{~kg}$ en las fincas relevadas.
Ordeño. Una rutina de ordeño completa debe incluir los procesos secuenciales que permitan minimizar las infecciones intramamarias, evitar la contaminación de la leche y lograr que el personal se encuentre cómodo con su trabajo. Además, la instalación se debe adaptar para disminuir el impacto del estrés calórico en los animales (Chang-Fung-Martel et al., 2017). Para lograr estos objetivos, el correcto dimensionamiento de la máquina de ordeño en relación a la cantidad de vacas y la funcionalidad de las instalaciones son fundamentales. El tiempo de ordeño depende, entre otros factores, del dimensionamiento de la máquina (cantidad de vacas por unidad de ordeño) y del diseño de la instalación de ordeño. Es necesario contar con una relación de $12 \mathrm{VO}$ por cada unidad de ordeño para lograr un trabajo rápido, en menos de 2 horas, para permitir a los operarios que trabajen con motivación y eficiencia (Jago y Burke, 2010).

En las fincas relevadas, el tiempo de ordeño completo excede los valores recomendados de 2 horas, siendo de 3,3 horas por ordeño, lo que puede impactar negativamente sobre el bienestar animal y la atención y motivación de los operarios durante el ordeño. A su vez, en comparación a los niveles de eficiencia de mano de obra logrados en otros países, los datos relevados muestran baja eficiencia de la mano de obra, con una persona cada $5( \pm 2)$ unidades de ordeño y $104( \pm 43,2)$ vacas por persona, para realizar la tarea de ordeño. La relación entre la cantidad de vacas y el total de operarios en la finca lechera dedicados al ordeño, crianza, alimentación y preparto es de 55,2 vacas por persona, valor superior al reportado en una reciente encuesta lechera argentina (INTA, 2018), pero bajo comparado con países eficientes, tales como Nueva Zelanda, donde se registran valores superiores a las 150 vacas por persona (Clark et al., 2007).

Respecto a la rutina de ordeño, en el 59\% de las fincas se realiza lavado de pezones en las vacas previo al ordeño, y de estos, el $62 \%$ procede luego a secarlos (el 38\% no los seca). En todos los casos se observó que se realiza "despunte" (eliminación manual de los primeros chorros de leche) previo al ordeño, y en el $62 \%$ de los casos se realiza sellado de los pezones post ordeño.

Se relevó que en el $83 \%$ de las fincas se realiza un servicio de mantenimiento programado de la máquina de ordeño; sin embargo, en un 35\% de los casos, este servicio se hace después de al menos un año o solo cuando hay algún problema, lo cual es inadecuado, debido a que se sugiere hacerlo con una frecuencia de 6 meses o menos.

Reproducción. En los establecimientos relevados el promedio de edad al primer parto fue de $29,( \pm 3,5)$ meses, sin embargo, el valor 
mínimo fue de 24,5 meses, lo que demuestra que es posible hacer más eficiente la recría de hembras. En todas las fincas relevadas se realiza inseminación artificial y en más del $86 \%$ se utiliza algún método de ayuda para la detección de celo (pinturas o parches). En cuanto al personal, el 71\% aprendió a inseminar por medio de algún curso de capacitación, mientras que el $29 \%$ restante aprendió de una manera informal.

Cultivo de alfalfa. Dada la importancia de este cultivo en la cadena forrajera de las fincas lecheras relevadas, es fundamental que el manejo de esta pastura sea el adecuado para maximizar su producción y aprovechamiento. Se relevó que el $100 \%$ de las fincas utilizó herbicidas de presiembra, importantes para garantizar mejores condiciones de competencia al momento de la implantación, y el $64 \%$ utilizó graminicidas. Solo en el 39\% de las fincas se hizo análisis de suelo para evaluar su fertilidad antes de la siembra. Pese a esto, en todos las fincas se fertilizan las pasturas de alfalfa, con valores promedio de 94 $\mathrm{kg} \mathrm{ha}^{-1} \mathrm{año}^{-1}$ de fertilizante total, valor superior al promedio de fertilización aplicado en Argentina (53 $\mathrm{kg} \mathrm{ha}^{-1}$ año ${ }^{-1}$; World Bank 2015), pero bajo en relación a las necesidades del cultivo en los suelos relevados. El bajo porcentaje de casos en los que se realiza muestras de suelo indica un bajo grado de profesionalización en la toma de decisiones sobre fertilización.

Debido a que solo en el 39\% de las fincas se analiza el suelo antes de implantar pasturas de alfalfa, se deduce que el desconocimiento del nivel nutricional del suelo limita la productividad de los forrajes y la productividad de leche en las fincas relevadas. El incremento de la fertilización permitiría incrementar la producción de forrajes en la región bajo estudio. Más aun, un estudio sobre cambio climático en dicha región reportó que se espera un incremento de las precipitaciones para las próximas décadas (Urcola et al., 2018), lo que haría aún más conveniente la fertilización de pasturas y cultivos, por mayor disponibilidad de agua.

Cultivo de maíz para silaje. En el cultivo de maíz, los niveles de fertilización realizados son elevados, con $214 \mathrm{~kg} \mathrm{ha}^{-1}$ de fertilizante promedio anual, pero solo se realiza análisis del suelo previo a la implantación del cultivo en el $41 \%$ de los casos.

Cultivo de invierno. Los cultivos de invierno más utilizados son raigrás anual (Lolium multiflorum) y avena (Avena sativa), en el $90 \%$ y $41 \%$ de las fincas, respectivamente. Estos cultivos son utilizados principalmente para pastoreo directo, y eventualmente para la confección de reservas. Todos los cultivos de invierno se fertilizan con $160 \mathrm{~kg} \mathrm{ha}^{-1}$ de fertilizante promedio al año.
Alimentación de las vacas en ordeño. En el $86 \%$ de las fincas relevadas se ofrece agua dentro del sector de alimentación. Los comederos para ofrecer alimento fuera de la sala de ordeño se observaron sanos y limpios en un $71 \%$ de los casos. En el $29 \%$ de los comederos se observó presencia de restos de alimentos, y en el 13\% de los casos esos residuos tenían mal olor, lo que podría influir en el consumo de alimentos (Favreau-Peigné et al., 2013).

Litros libres de alimentación. En promedio de las fincas se obtuvieron 6.957 $\mathrm{LLA} \mathrm{ha}^{-1} \mathrm{año}^{-1}$, lo que representa el 56,4\% de los $12.330 \mathrm{~L} \mathrm{ha}^{-1}$ año (productividad promedio). El desvío standard de la variable LLA ha-1 año-1 fue de 2.096, el máximo de 11.903 y el mínimo de 3.578 .

\section{Recursos naturales}

Los datos obtenidos de los análisis de agua y suelo realizados en el relevamiento se muestran en la Tabla 3.

Calidad de agua de bebida para ganado. El contenido de nitrato promedio relevado fue de $55( \pm 18) \mathrm{mg} \mathrm{kg}^{-1}$, superior al óptimo recomendado para el consumo animal, lo que podría comprometer la producción de leche y la reproducción de los animales (NRC, 2001). El resto de los parámetros evaluados presentó valores promedio considerados aptos para consumo de animales.

Calidad de suelo. Los resultados reflejaron niveles críticos de fósforo en el suelo, con un valor promedio de 9,3 $( \pm 6) \mathrm{mg} \mathrm{kg}^{-1}$. Para el cultivo de alfalfa se requieren suelos con más de $25 \mathrm{mg}$ $\mathrm{kg}^{-1}$ de fósforo, materia orgánica mayores al 3\% y $\mathrm{pH}$ cercanos a la neutralidad (Berone et al., 2017). Junto con el fósforo, el azufre constituye un elemento de gran importancia en la formación de las proteínas de la pastura y su oferta en el suelo guarda relación con los niveles de producción de materia seca. El nivel de sulfatos detectado fue en promedio de $11,7( \pm 2,5) \mathrm{mg} \mathrm{kg}^{-1}$, y en el $30 \%$ de los casos entre 7,9 y 9,8 $\mathrm{mg} \mathrm{kg}^{-1}$, por debajo de los $20 \mathrm{mg} \mathrm{kg}^{-1}$ recomendados (Vivas, 2006). El $72 \%$ de los suelos mostró un elevado contenido de materia orgánica, entre 3,2 y 6,0\%. El pH promedio fue de 6,6 , lo que representa un valor aceptable, ya que está dentro del rango de 6,5 a 7,5 en el que se encuentran los valores apropiados para el crecimiento de la mayoría de los cultivos (Sainz Rozas et al., 2011).

Análisis de correlaciones. Los resultados del análisis de correlación se muestran en la Tabla 4. $\mathrm{Si}$ bien se evaluaron las correlaciones entre todas las variables, solo se presentan las más relevantes.

En la Tabla 4 se observa que a medida que se incrementó la cantidad de VO por finca, se incrementó la cantidad de $\mathrm{VO}$ por unidad de 
Tabla 3. Calidad de suelo y calidad de agua en las fincas lecheras relevadas en la región noreste de la provincia de Buenos Aires, Argentina.

Table 3. Soil and water quality in surveyed dairy farms in the North East region of Buenos Aires province, Argentina.

\begin{tabular}{lcccc}
\hline & Promedio & Desvío standard & Max & Min \\
\hline & \multicolumn{4}{c}{ Agua } \\
pH & 7,4 & 0,20 & 7,5 & 7,0 \\
Bicarbonatos $\left(\mathrm{CO}_{3} \mathrm{H}^{-}\right), \mathrm{mg} \mathrm{kg}^{-1}$ & 543 & 132,7 & 757 & 347 \\
Carbonatos $\left(\mathrm{CO}_{3} \mathrm{H}^{-}\right), \mathrm{mg} \mathrm{kg}^{-1}$ & 463 & 410,5 & 665 & 163 \\
Sulfatos $\left(\mathrm{SO}_{4}^{=}\right), \mathrm{mg} \mathrm{kg}^{-1}$ & 31 & 71,6 & 164 & 4 \\
Cloruros $\left(\mathrm{CL}^{-}\right), \mathrm{mg} \mathrm{kg}^{-1}$ & 89 & 204,1 & 456 & 17 \\
Nitratos $\left(\mathrm{NO}_{3}^{-}\right), \mathrm{mg} \mathrm{kg}^{-1}$ & 55 & 18,4 & 78 & 24 \\
Sodio $\left(\mathrm{Na}^{+}\right), \mathrm{mg} \mathrm{kg}^{-1}$ & 61 & 11,1 & 70 & 48 \\
Total de sales, $\mathrm{mg} \mathrm{kg}^{-1}$ & 837 & 353,2 & 1679 & 521 \\
Residuos sólidos, mg kg-1 & 1068 & 420,9 & 1732 & 445 \\
& & & Suelo & \\
Materia orgánica, \% & 3,8 & 0,76 & 6,0 & 2,8 \\
Nitrógeno de nitratos, mg kg-1 & 18,1 & 4,82 & 31,6 & 10,5 \\
pH en agua $(1: 2,5)$ & 6,6 & 0,65 & 7,8 & 5,4 \\
Fósforo, mg kg-1 & 9,3 & 6,00 & 30,0 & 1,9 \\
Sulfatos, mg kg-1 & 11,7 & 2,45 & 17,0 & 7,9 \\
\hline
\end{tabular}

Tabla 4. Correlaciones entre las variables relevadas en 29 fincas lecheras en la Región Noreste de la provincia de Buenos Aires, Argentina.

Table 4. Correlations between surveyed variables in dairy farms of the North East region of Buenos Aires province, Argentina.

\begin{tabular}{llccc}
\hline Variable (1) & Variable (2) & n & $\begin{array}{c}\text { Correlación de } \\
\text { Pearson }\end{array}$ & Valor-p \\
\hline Cantidad VO & Cantidad VO unidades de ordeño & \\
Cantidad VO & Cantidad VO persona & 29 & $0,662^{* *}$ & 0.001 \\
Cantidad VO & Duración del ordeño (horas) & 29 & $0,782^{* *}$ & 0.001 \\
Cantidad VO & LLA ha & 29 & $0,785^{-1 *}$ & 0.001 \\
LLA ha $^{-1}$ & Superficie VT, ha & 17 & 0,216 & 0,405 \\
LLA ha $^{-1}$ & Carga animal, VT ha & 17 & 0,195 & 0,454 \\
LLA ha $^{-1}$ & Productividad anual, $\mathrm{L} \mathrm{ha}^{-1} \mathrm{año}^{-1}$ & 17 & $0,499^{*}$ & 0,041 \\
LLA ha $^{-1}$ & Producción por vaca, $\mathrm{LO}^{-1} \mathrm{día}^{-1}$ & 17 & $0,954^{* *}$ & 0,002 \\
LLA ha $^{-1}$ & Sombra natural, $\mathrm{m}^{2} \mathrm{VO}^{-1}$ & 17 & $0,582^{* *}$ & 0,014 \\
\hline
\end{tabular}

LLA: litros libres de alimentación

** $\mathrm{y}$ * Indican p-valores menores a 0,01 y 0,05 , respectivamente.

ordeño $(r=0,662)$ y el tiempo de ordeño $(r=0,785)$, lo que indica que las fincas con mayor cantidad de vacas tienen menor infraestructura de ordeño por $\mathrm{VO}$ y eso incrementa la demanda laboral, extendiendo el tiempo de ordeño. La cantidad de $\mathrm{VO}$ por persona $(\mathrm{r}=0,782)$ se incrementó con la cantidad de VO por finca, lo que sugiere mayor productividad de la mano de obra en fincas más grandes.
Otra variable de infraestructura, la sombra natural $\left(\mathrm{m}^{2} \mathrm{VO}^{-1}\right)$, presentó correlacion significativa con LLA ha-1, $(0,603)$, indicando que las fincas con más disponibilidad de sombra tendrían mejor resultado económico.

Se infiere, a partir del análisis de correlaciones, que las fincas que incrementaron la productividad a partir del incremento de la cantidad de vacas, aumentando el tamaño de los 
rodeos, no incrementaron en similar medida su infraestructura.

El tamaño de las fincas, medido a partir de superficie VT o cantidad VO no afectó el resultado económico medido a través de LLA ha ${ }^{-1}$ (Tabla 4), lo que sugiere que no existió un efecto de escala sobre el resultado económico.

La carga animal $\left(\mathrm{VT} \mathrm{ha}^{-1}\right)$, la productividad $\left(\mathrm{L} \mathrm{ha}^{-1}\right.$ año $\left.^{-1}\right)$ y la producción individual $\left(\mathrm{L} \mathrm{VO}^{-1}\right.$ $\left.\mathrm{dia}^{-1}\right)$, estuvieron positivamente relacionados al resultado económico (LLA ha ${ }^{-1}$ ), con coeficientes de correlación de 0,499, 0,954 y 0,582, respectivamente.

Implicancias del estudio. Si bien se observó un alto grado de asesoramiento agronómico y veterinario en las fincas lecheras relevadas, se encontraron en estado crítico aspectos esenciales para la producción de leche. Posiblemente, la mejora de las variables detectadas en estado crítico podría incrementar la producción de leche, mejorar la sustentabilidad y rentabilidad del negocio.

El diagnóstico y la información resultantes del presente estudio son herramientas útiles para definir proyectos de inversión conducentes a incrementar la productividad y la eficiencia productiva y económica de las fincas lecheras. La metodología de relevamiento presentada, podría ser de utilidad para diagnosticar limitantes en otras regiones lecheras de América del Sur.

\section{CONCLUSIONES}

Los principales aspectos críticos detectados en las fincas relevadas fueron: provisión de agua de bebida y sombras insuficientes, instalaciones de ordeño antiguas y sub-dimensionadas respecto al número de animales, lo que deriva en un exceso de horas de trabajo y puede impactar negativamente sobre el bienestar animal y la motivación de los operarios. También se detectó la carencia de nutrientes de suelo y falta de diagnóstico para mejorar fertilidad de los suelos.

En general, se observó que las fincas relevadas se encuentran alejadas de su estado óptimo en aspectos de infraestructura, estado de los suelos y el manejo general. Las inversiones tendientes a superar las limitantes detectadas podrían permitir incrementos en la producción de leche y mejorar la sustentabilidad y rentabilidad del negocio lechero.

\section{LITERATURA CITADA}

Adduci F., C. Labella, M. Musto, C. D'Adamo. and P. Freschi. 2015. Use of technical and economic parameters for evaluating cow ration efficiency. Italian Journal of Agronomy 10: 202-207.

Bareille, N., F. Beaudeau, S. Billon, A. Robert, and P. Faverdin. 2003. Effects of health disorders on feed intake and milk production in dairy cows. Livestock Production Science 83:53-56.

Baudracco, J, J. Maiztegui, J. Jáuregui, B. Lazzarini, R. Gagliardi, y A. Rosset. 2017. Productividad, resultado económico y riesgo de sistemas lecheros en el Centro-Norte de Argentina. Chilean Journal of Agricultural and Animal Sciences 72:454-466.

Baudracco, J., B. Lazzarini, A. Rosset, J. Jáuregui, D. Braida y J. Maiztegui. 2014. Cuantificación de limitantes productivas en tambos de Argentina. Reporte Final Proyecto INDICES. Disponible en http://www.infortambo.com/ admin/upload/arch/proyecto\%20indices.pdf (Consulta: 25 Mayo 2018).

Berone, G., E. Dinucci, H. Fernandez, L. Gastaldi, J. Mattera, y M. Spada. 2017. Calibración y validación de un modelo de crecimiento para alfalfa (Medicago sativa L.). Revista de Investigación Agropecuaria 43:256-265.

Cerqueira, J. Araújo, I. Blanco Penedo, J. Cantalapiedra, M. Silvestre, y S. Silva. 2016. Predicción de estrés térmico en vacas lecheras mediante indicadores ambientales y fisiológicos. Archivos de Zooteccnia 65:357364.

Chang-Fung-Martel, J., M.T. Harrison, R. Rawnsley, A.P. Smith, and H. Meinke. 2017. The impact of extreme climatic events on pasture-based dairy systems: a review. Crop and Pasture Science 68:1158-1169.

Chesterton, R.N., D.U. Pfeiffer, R.S. Morris, and C.M. Tanner. 1989. Environmental and behavioural factors affecting the prevalence of foot lameness in New Zealand dairy herds: a case-control study. New Zealand Veterinary Journal 37:135-142.

Chimicz, J., y E. Gambuzzi. 2007. Recientes cambios $\mathrm{y}$ posibles rumbos tecnológicos del tambo argentino. Revista Argentina de Producción Animal 27:322-323.

Clark, D.A., J.R. Caradus, R.M. Monaghan, P. Sharp, y B.S. Thorrold. 2007. Issues and options for future dairy farming in New Zealand. New Zealand Journal of Agricultural Research 50:203-221. 
Dikmen S. and P.J. Hansen 2009. Is the temperaturehumidity index the best indicator of heat stress in lactating dairy cows in a subtropical environment?, Journal of Dairy Science 92:109-116

Favreau-Peigné, A., R. Baumont, and C. Ginane. 2013. Food sensory characteristics: their unconsidered roles in the feeding behaviour of domestic ruminants. Animal 7:806-813.

Flamenbaum, I., and N. Gallon. 2010. Management of heat stress to improve fertility in dairy cows in Israel. Journal of Reproduction and Development 56:36-41.

INALE, 2018. Remisión a Planta INALE Uruguay 2018. Disponible en http://www.inale.org/ innovaportal/v/1963/4/innova.front/remisiona-planta.html (Consulta: 30 setiembre 2018).

INTA 2018. Informe de Lechería Pampeana del ejercicio 2016/2017. Disponible en https:// inta.gob.ar/documentos/lecheria-pampeanaresultados-productivos-ejercicio-2016-2017 (Consulta 30 setiembre 2018).

Jackson, M.L. 1982. Análisis químico de suelos. Omega, Barcelona, España.

Jago, J.G., and J.L.K. Burke. 2010. Shorter milking times. p. 132-148. In Proceedings of the South Island Dairy Event (SIDE) Conference, Invercargill, New Zealand.

Khan, M., D. Weary, and M. Von Keyserlingk. 2011. Invited review: effects of milk ration on solid feed intake, weaning, and performance in dairy heifers. Journal of Dairy Science 94:1071-1081.

Lazzarini, B., J. Baudracco, E. Demarchi, D. Lovino, y J.M. Jáuregui. 2014. Evolución de la suplementación, el consumo de pastura y la producción de leche en sistemas lecheros de Argentina. Revista FAVE - Ciencias Agrarias 13:73-78.

Lazzarini B., J. Baudracco, G. Tuñon, L. Gastaldi, N. Lyons, H. Quattrochi and N. LopezVillalobos. 2019. Review: Milk production from dairy cows in Argentina: Current state and perspectives for the future. Applied Animal Science 35:426-432.

Lawrence, K. E., R. N. Chesterton, and R. A. Laven. 2011. Further investigation of lameness in cows at pasture: an analysis of the lesions found in, and some possible risk factors associated with, lame New Zealand dairy cattle requiring veterinary treatment. Journal of Dairy Science 94:2794-805

LIC and DairyNZ. 2018. New Zealand Dairy Statistics 2017-18. Livestock Improvement Corporation (LIC) and Dairy NZ, Hamilton, New Zealand. Available at https://www. dairynz.co.nz/media/5790451/nz-dairystatistics-2017-18.pdf (Accessed 8 Nov. 2018).
World Bank. 2015. Fertilizer consumption. Available at https://data.worldbank.org (Accessed 2 Nov. 2018).

Mee, J.F. 2008. Newborn dairy calf management. Vet Clinic North America Food Animal Practice 24:1-17.

Miglierina M.M., N. Bonadeo, A.M. Ornstein, D. Becú-Villalobos, and I.M. Lacau-Mengido. 2018. In situ provision of drinking water to grazing dairy cows improves milk production. New Zealand Veterinary Journal 66:37-40.

NRC. 2001. National Research Council. Nutrient requirements of dairy cattle. 7th rev. ed. National Academy Press, Washington DC, USA.

OCLA. 2018. Observatorio de la Cadena Láctea Argentina. Disponible en http://www.ocla. org.ar (Consulta 30 setiembre 2018).

Sainz Rozas, H., H. Echeverria, y H. Angelini. 2011. Niveles de carbono orgánico y $\mathrm{pH}$ en suelos agrícolas de las regiones Pampeana y Extra Pampeana argentina. Ciencia del Suelo 29:29-37.

Sainz Rozas, H., H. Echeverria, y H. Angelini. 2012. Fósforo disponible en suelos agrícolas de la región Pampeana y Extra Pampeana argentina. Revista de Investigaciones Agropecuarias 38:33-39.

SENASA. 2018. Distribución de existencias bovinas por departamento. Disponible en https:// www.argentina.gob.ar/senasa/mercados-yestadisticas/estadisticas/animal-estadisticas/ bovinos/bovinos-y-bubalinos-sector-primario (Consulta 3 marzo 2018).

Torsein, M., A. Lindberg, C. Sandgren, K. Waller, M. Tornquist, and C. Svensson. 2011. Risk factors for calf mortality in large Swedish dairy herds. Preventive Veterinary Medicine 99:136-147.

Urcola, H.A., J.C. Burges, L. Gouarderes, and S. Solman. 2018. Climate change and cattle production: An evaluation for southeastern Buenos Aires, Argentina. Chilean Journal of Agricultural and Animal Sciences 34:254-465.

Veissier I, E. Van Laer, R. Palme, C.P.H. Moons, B. Ampe, B. Sonck, et al. 2018. Heat stress in cows at pasture and benefit of shade in a temperate climate region. International Journal of Biometeorology 62:585-595.

Vivas, H. 2006. Estrategias para la fertilización azufrada de alfalfa en un suelo del centro de Santa Fé. Informaciones Agronómicas del Cono Sur 30:12-15. Disponible en http://www.ipni.net/publication/ ia-lacs. (Consulta 30 septiembre 2018). 